\title{
Frequency of FLT3 Internal Tandem Duplications in Adult Syrian Patients with Acute Myeloid Leukemia and Normal Karyotype
}

\author{
Ismael F Al-Arbeed ${ }^{1 *}$, Abdulsamad Wafa ${ }^{2}$, Faten Moassass ${ }^{2}$, Bassel AL-Halabi $^{2}$, \\ Walid Al-Achkar², Imad Abou-Khamis' ${ }^{1}$
}

\begin{abstract}
Objective: Activating mutations of the fms-like tyrosine kinase 3 gene (FLT3) by internal tandem duplications (ITDs) in the juxtamembrane domain (JMD) have been reported in $\sim 30 \%$ of adult acute myeloid leukemia (AML) patients with cytogenetically normal karyotype (CN). However, FLT3/ITD mutations are frequently accompanied with leukocytosis, high percentage of blasts in bone marrow (BM), and increased the risk of treatment failure in AML patients. FLT3-ITD mutated AML patients mainly with normal karyotype have higher relapse probability and shorter duration of complete remission (CR) after chemotherapy, so FLT3-ITD mutation is considered as an independent poor prognostic factor in AML. Methods: FLT3-ITD and FLT3-KTD were studied by polymerase chain reaction (PCR) and restriction fragment length polymorphism- PCR (RFLP-PCR) in 44 adults AML patients with cytogenetically normal karyotype (AML-CN) at diagnosis to characterize FLT3 status. The results were correlated with the prognostic factors. Results: In this study, FLT3-ITD mutations were identified in 7 (15.9\%) of the 44 AML-CN patients. Among the 7 patients with FLT3/ITD mutations, 6 patients revealed a typical ITDs mutation (fragment size was 329 bp) and one patient showed untypical ITD mutation (fragment size was 400 bp). Whereas 37 patients $(61.7 \%)$ were FLT3-ITD. None of all AML-CN patients examined showed FLT3-KTD mutations. Conclusions: Our results support that FLT3-ITD are independent adverse prognostic factors for elderly AML-CN patients and are associated with low overall survival (OS), low rate of $\mathrm{CR}$, high relapse rate (RR), and high percentage of BM blast at diagnosis. We concluded, FLT3 mutation analysis should be performed as a routine test in AML-CN patients.
\end{abstract}

Keywords: Acute myeloid leukemia- normal karyotype- FLT3 internal tandem duplications- prognostic factors

Asian Pac J Cancer Prev, 22 (10), 3245-3251

\section{Introduction}

In patients with acute myeloid leukemia (AML) genetic diagnostics was performed in the past mainly by cytogenetics and molecular cytogenetics. In recent years also tumor markers were added, which can be uncovered only by molecular genetic methods (Kirtonia et al., 2020).

The fms-like tyrosine kinase 3 (FLT3) gene encodes a class III receptor tyrosine kinase for the FLT3 ligand, which is normally expressed in CD34+ hematopoietic stem/progenitor cells and plays a fundamental role in both normal and leukemic hematopoiesis (Stirewalt et al., 2003). Internal tandem duplications (ITDs) of the FLT3 gene (FLT3-ITDs) represent one of the most common molecular abnormalities in patients with AML, detectable in around $25 \%-30 \%$ of all patients (Schnittger et al., 2002; Patnaik 2018). ITDs consist of in-frame insertions of duplicated sequences localized in the juxtamembrane domain (JMD) of the FLT3 molecule. Their presence results in a constitutive, ligand independent activation of the tyrosine kinase activity of the FLT3 receptor; this is responsible for abnormal proliferation and differentiation of leukemia stem cells (Stirewalt et al., 2003). Constitutive activation of kinase domain is due to disruption of autoinhibitory interaction between JMD and the activation loop in AML, which normally stabilizes inactive kinase, and at the same time protects ATP binding pocket (Griffith et al., 2004; Chan 2011). Also, FLT3-ITDs protect leukemia cells from damaging chemotherapeutic agents (Lagunas-Rangel and Chávez-Valencia, 2017).

Presence of FLT3-ITDs has been associated with an increase an initial peripheral white blood cell (WBC) count, a high percentage of bone marrow blast cells, reduced disease-free survival (DFS) and overall survival (OS), and increased relapse rate (RR) with an overall adverse prognosis. However, rate of complete remission 
(CR) was not significantly affected (Kottaridis et al., 2001; Yanada et al. 2005; Canaani et al., 2018). Thus, a prognostic significance of FLT3/ITDs has been suggested (Stirewalt et al., 2006) and according to National Comprehensive Cancer Network and European LeukemiaNet (ELN) 2017, cases with FLT3-ITD mutation plus cytogenetically normal karyotype have a poor prognosis.

Whereas, the less frequent FLT3-TKD mutations are of unclear prognostic relevance have been observed in $\sim 7 \%$ of AML patients (Bacher et al., 2008; Yamamoto et al., 2001; Kim 2010). However, the incidence of ITD and TKD mutations in FLT3 vary slightly according to age, clinical risk groups, and cytogenetic profile (Levis, 2013). Moreover, adult AML patients usually have a higher prevalence of FLT3-ITDs than pediatric patients. This observation may partially explain why adult AML has a poorer clinical outcome than pediatric AML (Gregory et al., 2009). Many clinical studies have shown that patients with an ITD at diagnosis have frequent disease relapses and a short duration of survival when compared to patients without an ITD (Schnittger et al., 2002; Kim et al., 2004).

In this study, we investigated both the prevalence and prognostic significance of FLT3-ITDs in adult Syrian AML patients with cytogenetically normal (AML-CN) and could show, it has an impact on the therapy outcomes of AML.

\section{Materials and Methods}

\section{Subjects}

The present study comprised 44 newly patients diagnosed with de novo AML between October 2018 and February 2020 were included in this study. Patients without previous treatment were included in the study; patients with normal karyotype were selected for molecular analysis and patients with history of exposure to chemotherapy/radiotherapy, and secondary AML patients, were excluded. AML diagnosis was made according to French-American-British (FAB) classification. Their initial bone marrow (BM) or peripheral blood (PB) samples were collected for use in the study. Patients consisted of 23 men and 21 women; the median age was $35.3 \pm 12.4$ years (range, $18-77$ years) (Table 1 ). This study was approved by the Ethics Committee in Syrian Ministry of High Education and written informed consent was obtained from all the participants.

\section{Treatment protocol}

The majority of patients received $(3+7)$ standard induction chemotherapy, which consisted of daunorubicin at $45 \mathrm{mg} / \mathrm{m}^{2}$ for 3 days and cytarabine at $100-200 \mathrm{mg} / \mathrm{m} 2$ for 7 days, followed by high doses of a cytarabine-based consolidation phase (cytarabine at $\mathrm{mg} / \mathrm{m} 23$ every $12 \mathrm{~h}$ for 3 days, repeated for 2 to 3 cycles). Patients with acute promyelocytic leukemia (M3) received all-trans retinoic acid plus anthracycline. Patients received conventional induction chemotherapy and were followed for 14 months. BM aspiration was performed between 21 and 28 days after initiation of chemotherapy. The patients were followed up once every 3 months with clinical examination and complete blood counts. A BM aspiration was performed if there was any suggestion of relapse on clinical examination or peripheral smear.

\section{Cytogenetic and molecular cytogenetic analyses}

Chromosome analysis using GTG-banding was performed on BM sample prior to chemotherapy acc. to standard protocols (AL-Achkar et al., 2007). Fluorescence in situ hybridization (FISH) using specific probes to detect translocations $\mathrm{t}(8 ; 21), \mathrm{t}(15 ; 17), \mathrm{t}(16 ; 16), \mathrm{t}(12 ; 21)$, and deletion del(13q), were performed with standard method to excluded patients with chromosomal abnormalities, as previously reported (AL-Achkar et al., 2007).

\section{Sample collection}

Genomic DNA was isolated from PB or BM samples from de novo AML patients using the QIAamp DNA Blood Mini kit (Qiagen, Germany) according to the manufactures instructions and was stored at $-20^{\circ} \mathrm{C}$. The total DNA of each sample was measured by using a spectrophotometer followed by quantity ultraviolet light absorbance

\section{Analysis of the FLT3-ITD mutation}

Exons 14 and 15 of the FLT3-ITD mutation were amplified using specific forward primer 5'-GCAATTTAGGTATGAAAGCCAGC-3' and reverse primer 5'-CTTTCAGCATTTTGACGGCAACC-3' (Rezaei et al., 2017). The PCR reaction was performed in a total volume of $50 \mu$ l containing $200 \mathrm{ng}$ of genomic DNA, 10xPCR buffer (100 mM Tris- $\mathrm{HCl}, \mathrm{pH} 8.8,500$ $\mathrm{mM} \mathrm{KCl}), 2 \mathrm{mM} \mathrm{MgCl} 2,200 \mu \mathrm{M}$ dNTPs, $10 \mathrm{pM}$ of each primer, and $1 \mathrm{U}$ of Taq DNA polymerase. PCR conditions included initial denaturation at $95^{\circ} \mathrm{C}$ for $5 \mathrm{~min}$ followed by 30 cycles of $94^{\circ} \mathrm{C}$ for $30 \mathrm{~s}, 56^{\circ} \mathrm{C}$ for $30 \mathrm{~s}$, and $72^{\circ} \mathrm{C}$ for $45 \mathrm{~s}$ with a final extension at $72^{\circ} \mathrm{C}$ for $5 \mathrm{~min}$. PCR reaction was conducted in a PCR T100 thermocycler (Applied Biosystems, USA). The 329-bp PCR products were run

Table 1. Demographic and Laboratory Data of Syrian AML Cytogeneticlly Nnormal Patients

\begin{tabular}{lc}
\hline Parameters & Value \\
\hline Gender & $23(52.2 \%)$ \\
$\quad$ Male & $21(47.7 \%)$ \\
Female & 1.1 \\
Sex ratio (M/F) & $35.3 \pm 12.4$ \\
Age (median, range) & \\
FAB classification & $7(15.9 \%)$ \\
M1 & $8(18.1 \%)$ \\
M2 & $5(11.3 \%)$ \\
M3 & $13(29.5 \%)$ \\
M4 & $10(22.7 \%)$ \\
M5 & $1(2.2 \%)$ \\
M6 & $44(0.8-300)$ \\
WBC, x 10 $/ 1$ (median, range) & $8.7(3.5-16.7)$ \\
Hb, g/dl (median, range) & $78.3(17-309)$ \\
Plt, x 10\% $/ 1$ (median, range) & $69.6(42-94)$ \\
BM Blasts, $\%$ & W
\end{tabular}

FAB, French-American-British classifications; WBC, White blood cells; Hb, hemoglobin; Plt, Platelets; BM, bone marrow 
on 3\% agarose gel stained with DNA SafeStain Dye and visualized under UV light. Samples with additional longer PCR products were identified as FLT3-ITD+. All mutant samples were verified by direct sequencing using the ABI Prism 310 genetic analyzer (Applied Biosystems, Foster City, CA, USA). The cycle-sequencing reaction was performed in a $10-\mu \mathrm{l}$ volume containing $1 \mu \mathrm{l}$ of the terminator ready reaction, $5 \mathrm{pmol}$ of either the forward or reverse primer and $10 \mathrm{ng}$ of purified PCR product (ExoSAP-IT kit; Amersham BioSciences, Piscataway, NJ, USA). The thermal cycle protocol was $95^{\circ} \mathrm{C}$ for $4 \mathrm{~min}$ followed by 30 cycles at $96^{\circ} \mathrm{C}$ for $10 \mathrm{sec}, 50^{\circ} \mathrm{C}$ for $5 \mathrm{sec}$ and $60^{\circ} \mathrm{C}$ for $4 \mathrm{~min}$ (ABI GeneAmp PCR System 9700, Applied Biosystems). Centri-Sep columns (Princeton Separations, Adelphia, NJ, USA) were used for the effective and reliable removal of excess dye terminators (DyeEx 2.0, Qiagen, Germany) from completed DNA sequencing reactions. Data were compared and aligned with different sequences using the NCBI BLAST Assembled Genomes tool (http://blast.ncbi.nlm.nih.gov/ Blast.cgi).

\section{Analysis of the FLT3-TKD mutation}

For detection of the FLT3-TKD mutation, the specific forward primer 5'-CCGCCAGGAACGTGCTTG-3' and reverse primer 5'-GCAGCCTCACATTGCCCC-3' were used (Rezaei et al., 2017). The PCR reaction was performed in a total volume of $15 \mu \mathrm{l}$ with similar reagents as used for the FLT3-ITD mutation, except for the primers. PCR conditions were also the same, except for the annealing temperature, which was $65^{\circ} \mathrm{C}$ for 30 $\mathrm{s}$. The amplification reaction was conducted in a PCR T100 thermocycler (Applied Biosystems). The 119-bp PCR products were then digested with $2 \mathrm{U}$ of EcoRV at $37^{\circ} \mathrm{C}$ for $17 \mathrm{~h}$, run on $3 \%$ agarose gel stained with DNA SafeStain Dye, and visualized under UV light. The presence of an undigested PCR product was an indication of a mutant sample.

\section{Statistical analysis}

The comparison of qualitative data such as age, WBC count, platelet count, hemoglobin level and blast count percentage between FLT3-ITD+ and FLT3-ITD- patients were statistically evaluated using Fisher exact and chisquare tests. OS and DFS were estimated for patients who received at least one induction course of therapy using the Kaplan-Meier method. $\mathrm{p}<0.05$ was considered to be of statistical significance. All analyses were performed using SPSS Statistics 19 software (SPSS, Chicago, IL, USA).

\section{Results}

Table 2 summarizes the characteristics of the newly diagnosed AML-CN patients included in the study. Of the 44 AML patients studied, 23 were males (52.2\%) and 21 were females $(47.7 \%) ; 7$ out 44 cases ( 3 males and 4 females) were positive for FLT3-ITD+ mutations $(15.9 \%), 6$ out 7 FLT3-ITD+ patients (fragment size was more than 329 bp) and one out 7 FLT3/ITD+ patients showed untypical ITDs mutation (fragment size was $\sim 400$ bp) (Alarbeed et al., 2021); whereas 37 patients (61.7\%) were FLT3-ITD- (fragment size was 329 bp). None of all AML-CN patients examined showed FLT3-KTD mutations.

There were no significant differences between FLT3-ITD+ and FLT3-ITD-patients with respect to sex, WBC, hemoglobin, platelet counts, and AML FAB subtypes (Table 2). However, patients with FLT3-ITD+ was older than FLT3-ITD- patients $(47.9 \pm 14.4$ vs. $32.8 \pm 10.4 ; \mathrm{p}=0.002$ ) and had high percentage of bone marrow blasts compared with FLT3-ITD- patients ( $78.3 \pm 13.9$ vs. $67.3 \pm 13.6 ; p=0.07)$.

Of the 44 patients who received standard induction chemotherapy, 39 patients $(88.6 \%)$ achieved CR. The CR rate was significantly affected in patients with FLT3-ITD+ compared FLT3-ITD- (28.6\% vs. $75.6 \%$, p<0.01) (Table 3 ). However, of the patients who achieved CR, patients with FLT3-ITD+ had a higher RR and a low OS than patients with FLT3-ITD- (RR: $57.1 \%$ vs. $13.5 \%$, $\mathrm{p}=<0.01$ ), (OS rate: 4 vs. 8.2 months, $\mathrm{p}=0.03$ ) (Table 4, Figure 1). However, these differences were statistically significant.

Table 2. Clinical Patients Characteristics According to FLT-3 Status in Syrian AML Normal Cytogeneticlly Patients

\begin{tabular}{|c|c|c|c|}
\hline Features & FLT3-ITD+ & FLT3-ITD- & $\mathrm{P}$ value \\
\hline Patients no (\%) & $7(15.9 \%)$ & $37(48.09 \%)$ & \\
\hline \multicolumn{4}{|l|}{ Gender } \\
\hline Male & $3(42.9 \%)$ & $20(54 \%)$ & 0.6 \\
\hline Female & $4(57.1 \%)$ & $17(46 \%)$ & \\
\hline Sex ratio $(M / F)$ & 0.75 & 1.1 & \\
\hline \multicolumn{4}{|l|}{ Age (years) } \\
\hline Mean & $47.9 \pm 14.4$ & $32.8 \pm 10.4$ & 0.002 \\
\hline range & $18-64$ & $18-57$ & \\
\hline \multicolumn{4}{|l|}{ WBC, $x 10^{9} / 1$} \\
\hline Median & $41.8 \pm 21.3$ & $44.6 \pm 65.1$ & 0.9 \\
\hline Range & $4.5-64$ & $0.8-300$ & \\
\hline \multicolumn{4}{|l|}{$\mathrm{Hb}(\mathrm{g} / \mathrm{dl})$} \\
\hline Median & $8.6 \pm 1.4$ & $8.7 \pm 2.6$ & 0.9 \\
\hline Range & $6-10.5$ & $3.5-16.7$ & \\
\hline \multicolumn{4}{|l|}{ Plt $\times 10^{9} / 1$} \\
\hline Median & $81.4 \pm 29.6$ & $77.5 \pm 55.9$ & 0.9 \\
\hline Range & $32-112$ & $17-309$ & \\
\hline \multicolumn{4}{|l|}{ BM Blasts, $\%$} \\
\hline Median & $78.3 \pm 13.9$ & $67.3 \pm 13.6$ & 0.07 \\
\hline Range & $60-94$ & $42-90$ & \\
\hline FAB:M4\&M5/others & $4(57.1 \%)$ & $19(51.3 \%)$ & 0.8 \\
\hline
\end{tabular}

FAB, French-American-British classifications; WBC, White blood cells; $\mathrm{Hb}$, hemoglobin; Plt, Platelets; $\mathrm{BM}$, bone marrow. $\mathrm{P}<0.05$ is considered significant

Table 3. Outcome Data According to FLT/ITD Mutational Status in Syrian AML-CN Patients

\begin{tabular}{|c|c|c|c|c|}
\hline \multirow[t]{2}{*}{ Groups } & \multicolumn{3}{|c|}{ Fate } & \multirow[t]{2}{*}{$P$} \\
\hline & $\mathrm{CR}$ & $\mathrm{RD}$ & ID & \\
\hline FLT3-ITD+ & $2 / 7(28.6 \%)$ & $4 / 7(57.1 \%)$ & $1 / 7(14.3 \%)$ & $<0.01$ \\
\hline FLT3-ITD- & $28 / 37(75.6 \%)$ & $5 / 37(13.5 \%)$ & $4 / 37(10.8 \%)$ & \\
\hline
\end{tabular}

$\mathrm{CR}$, complete remission; $\mathrm{RD}$, resistant disease; ID, induction death.

Asian Pacific Journal of Cancer Prevention, Vol $22 \quad \mathbf{3 2 4 7}$ 


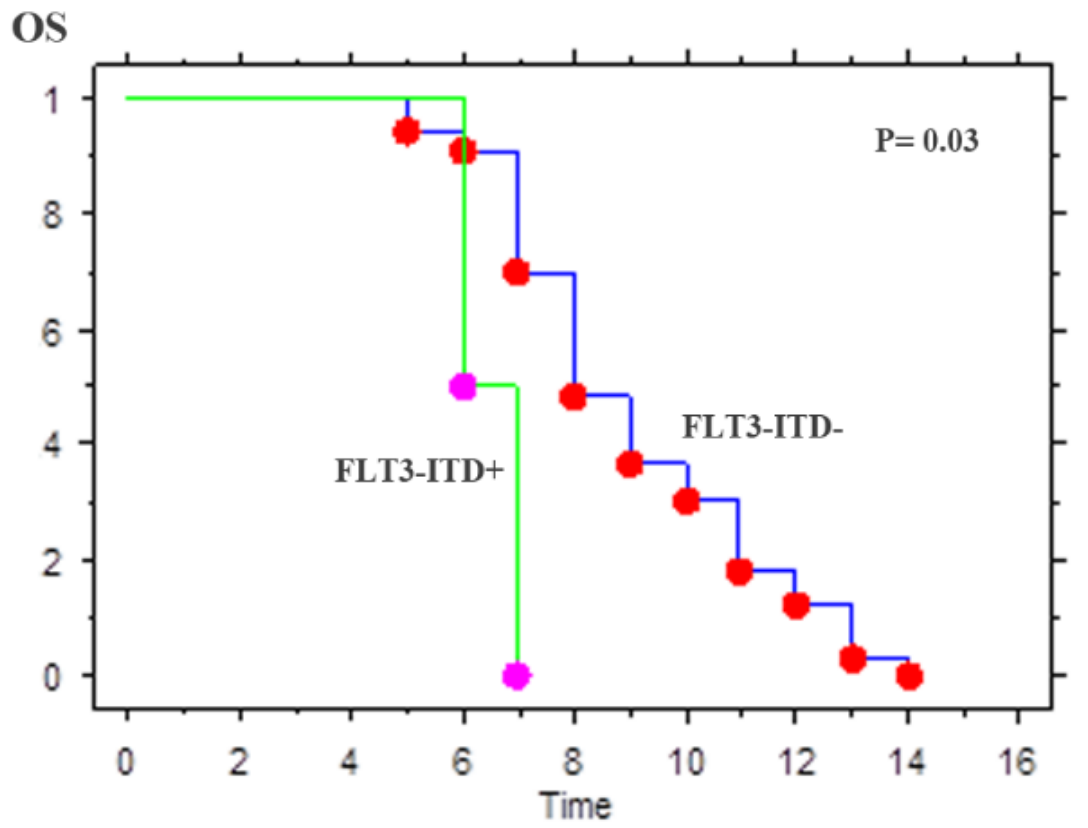

Figure 1. Kaplan-Meier AAnalysis of OS of Patients with AML- CN According to the FLT3-ITD Status

Table 4. Outcome Data According to FLT/ITD Mutational Status in Syrian AML-CN Patients

\begin{tabular}{lcccc}
\hline Mutational status & $\begin{array}{c}\text { Mean OS } \\
\text { (months) }\end{array}$ & $\mathrm{P}$ & $\begin{array}{c}\text { Mean DFS } \\
\text { (months) }\end{array}$ & $\mathrm{P}$ \\
\hline FLT3-ITD- & 8.2 & 0.03 & 8.8 & 0.02 \\
FLT3-ITD+ & 4.0 & & 3.3 & \\
\hline
\end{tabular}

OS, overall survival; DFS, disease-free survival

FLT3-ITD+ was a sole independently poor prognostic factor for DFS and OS.

\section{Discussion}

We evaluated the prevalence and prognostic FLT3-ITD+ in 44 Syrian adult patients with AML-CN newly diagnosed.

The presence of FLT3-ITD+ had received significant attention due to contribute to disease progression and poor prognosis for patients. The clinical significance of FLT3-ITD+ has been clearly demonstrated in previous studies (Levis, 2013; Thiede et al., 2002). Adult studies have shown a prevalence of $25-30 \%$ for the FLT3-ITD+ in AML patients who have no cytogenetic abnormalities (Schnittger et al., 2002; Patnaik 2018; Griffith et al., 2004) and $\sim 7 \%$ for FLT3-KTD point mutation of the activation loop domain (Bacher et al., 2008; Yamamoto et al., 2001; Kim 2010). The incidence of FLT3-ITD+ in the current study was $15.9 \%$, which is obviously similar incidences having been previously reported in some studies (Table 5) (Elyamany et al., 2014; Aly et al., 2012; Pazhakh et al., 2011). Several studies observed a lower

Table 5. Prevalence of FLT3-ITD Mutation in AML in Various Studies

\begin{tabular}{lccc}
\hline Study & Country & Number of AML cases & Total prevalence \% \\
\hline Present study & Syria & 44 & 15.9 \\
Thiede et al. (2002) & Germany & 979 & 20.4 \\
Elyamany et al. (2014) & Saudi Arabia & 97 & 14.4 \\
Aly et al. (2012) & Egypt & 39 & 15.4 \\
Pazhakh et al. (2011) & Iran & 131 & 16 \\
Ishfaq et al. (2012) & Pakistan & 30 & 13.3 \\
Gari et al. (2008) & Saudi Arabia & 129 & 11.6 \\
Sheikhha et al. (2003) & Iran & 80 & 10 \\
Xu et al. (2012) & China & 216 & 20.8 \\
Wang et al. (2005) & China & 76 & 19.7 \\
Al-Tonbary et al. (2009) & Egypt & 30 & 20 \\
Suzuki et al. (2007) & Japan & 60 & 20 \\
Wang et al. (2010) & China & 143 & 25.9 \\
Auewarakul et al. (2005) & Thiland & 256 & 27.3 \\
Fröhling et al. (2002) & USA & 224 & 32 \\
\hline
\end{tabular}


incidence of FLT3-ITD + than in our study (Elyamany et al., 2014; Aly et al., 2012; Pazhakh et al., 2011; Ishfaq et al., 2002; Gari et al., 2008; Sheikhha et al., 2003). Various studies have detected a high occurrence of FLT3-ITD+ (Thiede et al., 2002; Xu et al., 2012; Wang et al., 2010; Al-tonbary et al., 2009; Suzuki et al., 2007; Wang et al., 2005; Auewarakul et al., 2005; Fröhling et al., 2002). However, those studies evaluated AML with or without abnormal karyotype. The differences in these results may additionally be explained due to sample sizes, geographic and ethnic background of the studied populations.

The clinical impact of the characteristics of patients with and/or without FLT3-ITD demonstrated no statistically significant difference between FLT3-ITD+ and FLT3-ITD- patients regarding to a gender, our findings which are an agreement with those have been reported (Bao et al., 2006; Zwaan et al., 2003). Clinically, AML patients with FLT3-ITD+ tend to have higher WBC counts and an increased percentage of leukemic blasts (Kottaridis et al., 2001; Yanada et al., 2005; Canaani et al., 2018). In our study, a positive association has been found between FLT3-ITD+ mutation versus BM blasts count (Kottaridis et al., 2001; Yanada et al., 2005; Canaani et al., 2018).

Regarding to CR rate, presence of the FLT3-ITD+ mutation did not appear to influence the achievement of CR (Kottaridis et al., 2001; Yanada et al., 2005; Canaani et al., 2018). In the other hand, many studies have demonstrated that FLT3-ITD+ patients had low CR rate, short OS, and high cumulative incidence of relapse after chemotherapy combined with FLT3 inhibition (Chen et al., 2020; Meshinchi et al., 2001; Al-Mawali A et al., 2013). In our study, CR rate was statistically lower in FLT3-ITD+ $(28.6 \%)$ than that in FLT3-ITD- $(78.4 \%)$ subjects (P $<0.01$ ) our results in agreement with previously findings (Chen et al., 2020; Meshinchi et al., 2001; Al-Mawali A et al., 2013).

Although the clinical significance of this FLT3 mutation especially in AML-CN not clear yet. However, several studies showed that FLT3-ITD+ mutation is a strong adverse prognostic factor in AML patients (Stirewalt et al., 2008; Kiyoi and Naoe 2006; Zheng and Small 2005) with reduced DFS and OS and increased RR (Kottaridis et al., 2001; Yanada et al., 2005; Canaani et al., 2018). In our study, median OS was 4.0 months for FLT3-ITD+ patients and 8.2 months for FLT3-ITD- patients ( $p=0.03$ ), and DFS was also worse for FLT3-ITD+ than FLT3-ITD- patients ( $p=0.02)$ because of a higher RR in FLT3-ITD+ patients. Also, high leucocytes count, high blast cells count in peripheral blood and resistance to therapy confers a poor prognosis. This has led to the development of a number of small molecule tyrosine kinase inhibitors (TKI) with activity against FLT3 (Small 2006; Leung et al., 2013). Moreover, patients with low or absent levels of FLT3-ITD, consistent with homozygosity for the FLT3-ITD allele, appear to have a particularly dismal outcome (Thiede et al., 2002).

A failure to achieve post-induction remission was observed in $57.1 \%(4 / 7)$ of evaluable pediatric patients with FLT3-ITD+, as opposed to $13.5 \%$ (5/37) of FLT3-ITD- patients $(\mathrm{P}=<0.01)$, our findings in accordance with Kumiko et al. (Kumiko et al., 2005). Most of patients with FLT3-ITD+ were found to be resistant to initial chemotherapy and failed to achieve CR (Xu et al., 2000; Arrigoni et al., 2003). Older patients (age less than 60 years) with FLT3-ITD+ mutation have a significantly association with increased RR (Kottaridis et al., 2003).

Schnittger et al., (2018) could show that decreased FLT3-ITDs positively correlates with older age. However, other studies that did not reveal any age-dependency of FLT3-ITD, being performed in cohorts including other cytogenetic groups, smaller patient numbers of AML-CN, or restriction to patients up to 60 years of age (Thiede et al., 2002; Fröhling et al., 2002; Gale et al., 2005). In our study, patients who had FLT3-ITD+ were older than FLT3-ITD- $(\mathrm{P}=0.002)$.

In Conclusion, we report here for the first time the frequency and prognosis of the presence of FLT3-ITD+ mutations in adult Syrian patients with AML-CN. The frequency of FLT3 mutations in our study was lower $(15.9 \%)$ than in previous studies; however, some reports agree with our observation, and that these mutations are an important adverse prognostic factor. Overall, this report supports the view that FLT3-ITD+ is a strong prognostic factor in AML patients and is associated with low CR, high $\mathrm{RR}$, resistance to therapy, low OS, low DFS and confers a poor prognosis. Thus, FLT3 mutation analysis should be performed as a routine test in AML-CN patients.

\section{Author Contribution Statement}

IA provided all cases, a clinical data and a chemotherapy plan; AW and WA did primary cytogenetic and main part of the FISH-tests; FA and BA performed the molecular cytogenetic analyses; IA scientific supervisor of the IA student and put of the work plan. IA and AW drafted the paper and all authors worked on the final version of the paper. All authors read and approved the final manuscript.

\section{Acknowledgements}

None.

\section{Ethics approval}

This study was approved by the Ethics Committee in Syrian Ministry of High Education and written informed consent was obtained from all the participants.

\section{Availability of data}

All data generated or analyzed during this study are included in this published article.

\section{Competing interests}

The author(s) declare that they have no competing interests.

\section{References}

Alarbeed IF, Wafa A, Moassass F, et al (2021). De novo adult acute myeloid leukemia with two new mutations in juxtatransmembrane domain of the FLT3 gene- A case report. J Med Case Rep, 15, 22.

Asian Pacific Journal of Cancer Prevention, Vol 22 
AL-Achkar W, Wafa A, Nweder MS (2007). A complex translocation $\mathrm{t}(5 ; 9 ; 22)$ in Philadelphia cells involving the short arm of chromosome 5 in a case of chronic myelogenous leukemia. J Exp Clin Cancer Res, 26, 411-5.

Al-Mawali A, Gillis D, Lewis I (2013). Characteristics and prognosis of adult acute myeloid leukemia with internal tandem duplication in the FLT3 gene. Oman Med J, 28, 432-40.

Al-tonbary Y, Mansour AK, Ghazy H, et al (2009). Prognostic significance of foetallike tyrosine kinase 3 mutation in Egyptian children with acute Leukaemia. Inter J Lab Hemat, 3, 320-6.

Aly R, Shahin D, Azmy E (2012). Prognostic significance of FLT3 internal tandem duplication in Egyptian acute myeloid leukemia and normal cytogenetics. Comp Clin Pathol, 21, 1029-35.

Arrigoni P, Beretta C, Silvestri D, et al (2003). FLT3 internal tandem duplication in childhood acute myeloid leukaemia: association with hyperleucocytosis in acute promyelocytic leukaemia. Br J Haematol, 120, 89-92.

Auewarakul CU, Sritana N, Limwongse C, et al (2005). Mutations of the FLT3 gene in adult acute myeloid leukemia: determination of incidence and identification of a novel mutation in a Thai population. Cancer Genet Cytogenet, 162, 127-34.

Bacher U, Haferlach C, Kern W, et al (2008). Prognostic relevance of FLT3-TKD mutations in AML: the combination matters - an analysis of 3082 patients. Blood, 111, 2527-37.

Bao L, Wang X, Ryder J, et al (2006). Prospective study of 174 de novo acute myelogenous leukemias according to the WHO classification: subtypes, cytogenetic features and FLT3 mutation. Eur J Haematol, 77, 35-45.

Canaani J, Labopin M, Huang XJ, et al (2018). T-cell replete haploidentical stem cell transplantation attenuates the prognostic impact of FLT3-ITD in acute myeloid leukemia: a report from the Acute Leukemia Working Party of the European Society for Blood and Marrow Transplantation. Am J Hematol, 93, 736-44.

Chen F, Sun J, Yin C, et al (2020). Impact of FLT3-ITD allele ratio and ITD length on therapeutic outcome in cytogenetically normal AML patients without NPM1 mutation. Bone Marrow Transplant, 55, 740-8.

Chan PM (2011). Differential signaling of Flt3 activating mutations in acute myeloid leukemia: a working model. Protein Cell, 2, 108-15.

Elyamany G, Awad M, Fadalla K, et al (2014). Frequency and Prognostic Relevance of FLT3 Mutations in Saudi Acute Myeloid Leukemia Patients. Adv Hematol, 2014, 141360.

Kim HJ(2010). Mutations in AML with a normal karyotype: NPM1 and FLT3-ITD, ready to use as a key prognosticator?. Korean $J$ Hematol, 45, 79-80.

Kim YK, Lee JJ, Lee YR, et al (2004). The presence of FLT3/ ITD mutations is an independent prognostic factor in acute myeloid leukemia patients with normal karyotype. Blood, 104, 3008.

Kiyoi H, Naoe T (2006). Biology, clinical relevance, and molecularly targeted therapy in acute leukemia with FLT3 mutation. Int $J$ Hematol, 83, 301-8.

Kirtonia A, Pandya G, Sethi G, et al (2020). A comprehensive review of genetic alterations and molecular targeted therapies for the implementation of personalized medicine in acute myeloid leukemia. J Mol Med (Berl), 98, 1069-91.

Kumiko O, Sakurako Y, Sumio K, et al (2005). Activating mutations of FLT3 gene in childhood acute myeloid leukemia and their applications for detection of minimal residual disease. Japan J Pediatr Hematol, 19, 19-24.

Kottaridis PD, Gale RE, Frew ME, et al (2001). The presence of a FLT3 internal tandem duplication in patients with acute myeloid leukemia (AML) adds important prognostic information to cytogenetic risk group and response to the first cycle of chemotherapy: analysis of 854 patients from the United Kingdom Medical Research Council AML 10 and 12 trials. Blood, 98, 1752-9.

Kottaridis P, Gale RE, Linch DC (2003). FLT3 mutations and leukemia. Br J Haematol, 122, 523-538.

Fröhling S, Schlenk RF, Breitruck J, et al (2002). Prognostic significance of activating FLT3 mutations in younger adults (16 to 60 years) with acute myeloid leukemia and normal cytogenetics: a study of the AML Study Group Ulm. Blood, 100, 4372-80.

Gale RE, Hills R, Pizzey AR, et al (2005). Relationship between FLT3 mutation status, biologic characteristics, and response to targeted therapy in acute promyelocytic leukemia. Blood, 106, 3768-76.

Gari M, Abuzenadah A, Chaudhary A, et al (2008). Detection of FLT3 oncogene mutations in acute myeloid leukemia using conformation sensitive gel electrophoresis. Int J Mol Sci, 9, 2194-2204.

Gregory TK, Wald D, Chen Y, et al (2009). Molecular prognostic markers for adult acute myeloid leukemia with normal cytogenetics. J Hematol Oncol, 2, 23.

Griffith J, Black J, Faerman C, et al (2004). The structural basis for autoinhibition of FLT3 by the juxtamembrane domain. Mol Cell, 13, 169-78.

Ishfaq M, MalikA, Faiz M, et al (2012). Molecular characterization of FLT3mutations in acute leukemia patients in Pakistan. Asian Pac JCancer Prev, 13, 4581-5.

Lagunas-Rangel FA, Chávez-Valencia V (2017). FLT3-ITD and its current role in acute myeloid leukaemia. Med Oncol, 34, 114.

Leung AYH, Man C-H, Kwong Y-L (2013). FLT3 inhibition: a moving and evolving target in acute myeloid. Leukemia, 27, 260-8.

Levis M (2013). FLT3 mutations in acute myeloid leukemia: what is the best approach in 2013?. Hematol Am Soc Hematol Educ Program, 2013, 220-6.

Meshinchi S, Woods WG, Stirewalt DL, et al (2001). Prevalence and prognostic significance of Flt3 internal tandem duplication in pediatric acute myeloid leukemia. Blood, 97, 89-94.

Patnaik MM (2018). The importance of FLT3 mutational analysis in acute myeloid leukemia. Leuk Lymphoma, 59, 2273-86.

Pazhakh V, Zaker F, Alimoghaddam K, et al (2011). Detection of nucleophosmin and FMS-like tyrosine kinase-3 gene mutations in acute myeloid leukemia. Ann Saudi Med, 31, $45-50$.

Rezaei N, Arandi N , Valibeigi B, et al (2017). FMS-Like Tyrosine Kinase 3 (FLT3) and Nucleophosmin 1 (NPM1) in Iranian Adult Acute Myeloid Leukemia Patients with Normal Karyotypes: Mutation Status and Clinical and Laboratory Characteristics. Turk J Haematol, 34, 300-6.

Schnittger S, Schoch C, Dugas M, et al (2002). Analysis of FLT3 length mutations in 1003 patients with acute myeloid leukemia: correlation to cytogenetics, FAB subtype, and prognosis in the AMLCG study and usefulness as a marker for the detection of minimal residual disease. Blood, 100, 59-66.

Sheikhha MH, Awan A, Tobal K, et al (2003). Prognostic significance of FLT3 1TD and D835 mutations in AML patients. Hematol J, 4, 41-6.

Small D (2006). FLT3 mutations: biology and treatment. Hematol Am Soc Hematol Educ Program, 2006, 178-84.

Stirewalt DL, Kopecky KJ, Meshinchi S, et al (2006). Size of FLT3 internal tandem duplication has prognostic significance 
in patients with acute myeloid leukemia. Blood, 107, 3724-6.

Stirewalt DL, Radich JP (2003). The role of FLT3 in haematopoietic malignancies. Nat Rev Cancer, 3, 650-65.

Suzuki R, Onizuka M, Kojima M, et al (2007). Prognostic significance of FLT3 internal tandem duplication and NPM1mutations in acute myeloid leukemia in an unselected patient population. Int J Hematol, 86, 422-8.

Thiede C, Steudel C, Mohr B, et al (2002). Analysis of FLT3activating mutations in 979 patients with acute myelogenous leukemia: association with FAB subtypes and identification of subgroups with poor prognosis. Blood, 99, 4326-35.

Wang L, Lin D, Zhang X, et al (2005). Analysis of FLT3 internal tandem duplication and D835 mutations in Chinese acute leukemia patients. Leuk Res, 29, 1393-8.

Wang Y, Li Z, He C, et al (2010). MicroRNAs expression signatures are associated with lineage and survival in acute leukemias. Blood Cells Mol Dis, 44, 191-7.

Xu F, Taki T, Eguchi M, et al (2000). Tandem duplication of the FLT3 gene is infrequent in infant acute leukemia. Japan Infant Leukemia Study Group. Leukemia, 14, 945-947.

Xu YY, Gao L, Ding Y, et al (2012). Detection and clinical significance of FLT3-ITD gene mutation in patients with acute myeloid leukemia. Zhongguo Shi Yan Xиe Ye Xиe Za Zhi, 20, 1312-5.

Yamamoto Y, Kiyoi H, Nakano Y, et al (2001). Activating mutation of D835 within the activation loop of FLT3 in human hematologic malignancies. Blood, 97, 2434-9.

Yanada M, Matsuo K, Suzuki T, et al (2005). Prognostic significance of FLT3 internal tandem duplication and tyrosine kinase domain mutations for acute myeloid leukemia: a meta-analysis. Leukemia, 19, 1345-9.

Zheng R, Small D (2005). Mutant FLT3 signaling contributes to a block in myeloid differentiation. Leuk Lymphoma, 46, 1679-87.

Zwaan CM, Meshinchi S, Radich JP, et al (2003). FLT3 internal tandem duplication in 234 children with acute myeloid leukemia: prognostic significance and relation to cellular drug resistance. Blood, 102, 2387-94.

This work is licensed under a Creative Commons AttributionNon Commercial 4.0 International License. 